Cahiers de recherches médiévales

Journal of medieval studies

15 | 2008

La Tentation du parodique dans la littérature médiévale

\title{
Et succurere Terre sancte pro posse
}

Les Templiers castillans et la défense de l'Orient latin au tournant des $\mathrm{XIII}^{\mathrm{e}}$ et $\mathrm{XIV}^{\mathrm{e}}$ siècles

Philippe Josserand

\section{(2) OpenEdition}

\section{Journals}

Édition électronique

URL : https://journals.openedition.org/crm/5792

DOI : $10.4000 / \mathrm{crm} .5792$

ISSN : $1955-2424$

Éditeur

Honoré Champion

Édition imprimée

Date de publication : 20 juin 2008

Pagination : 217-235

ISSN : $1272-9752$

Référence électronique

Philippe Josserand, «Et succurere Terre sancte pro posse », Cahiers de recherches médiévales [En ligne], 15 | 2008, mis en ligne le 20 juin 2011, consulté le 15 décembre 2022. URL : http:// journals.openedition.org/crm/5792; DOI : https://doi.org/10.4000/crm.5792 


\title{
ब飘M
}

\section{Et succurere Terre sancte pro posse : les Templiers castillans et la défense de l'Orient latin au tournant des $\mathrm{XIII}^{\mathrm{e}}$ et $\mathrm{XIV}^{\mathrm{e}}$ siècles*}

\begin{abstract}
The implantation in the Eastern Mediterranean of the military orders from the Hispanic West has seldom been studied since for most scholars, be they ancient or modern, the brethren of these institutions are thought to have acted in a merely national sense. In spite of its investment in Syria until its suppression, the Temple - just like other military orders does not escape to such a perspective. Despite being scattered and rare, the sources show that Templars from Castile and León had fought in greater proportion than one usually contends in order to protect the Latin East and, after 1291 on to try and recover it. From the well-known brother Reddecœur, a close collaborator of Guillaume de Beaujeu and one of the unfortunate defenders of Tripoli in 1289, whose Asturian origin is to be noted, to the provincial master Rodrigo Yánez, whose passage overseas is testified in 1305, one could assess the human and material investment of a remote Templar land whose relations with the Latin East deserve to be better approached and should stop being underestimated, if not, even worse, ignored altogether.
\end{abstract}

Résumé : Rarement l'implantation en Méditerranée orientale des ordres militaires issus de l'Occident hispanique a été prise en compte dans l'historiographie car, pour beaucoup d'auteurs anciens mais également modernes, leurs frères sont réputés agir dans un cadre qui serait avant tout national. Pas plus que ses pairs, le Temple, pourtant étroitement lié à la Syrie jusqu'à sa chute tragique, n'a échappé à un tel regard. Or les sources manifestent, en dépit de la difficulté liée à leur éclatement et à leur parcimonie, que des membres de l'ordre originaires des royaumes de Castille et de León ont combattu en plus grand nombre qu'on ne l'estime à l'ordinaire pour défendre l'Orient latin et, après 1291, pour tenter de le recouvrer. Du célèbre frère Reddecour, proche de Guillaume de Beaujeu et défenseur malheureux de Tripoli en 1289, auquel il convient de restituer son origine asturienne, au maître provincial Rodrigo Yáñez, dont le passage outre-mer est attesté en 1305, leur exemple révèle l'investissement humain et financier de terres templières certes lointaines, mais dont les liens avec l'Orient latin, qui mériteraient d'être mieux étudiés, doivent d'ores et déjà cesser d'être tenus pour négligeables ou, pire encore, pour inexistants.

L'implantation en Méditerranée orientale des ordres militaires issus de l'Occident hispanique a rarement été prise en compte dans l'historiographie car, pour beaucoup d'auteurs anciens mais également modernes, ils passent pour avoir

\footnotetext{
* Ce texte a été écrit pour le $V$ Encontro sobre ordens militares. As ordens militares e as ordens de cavalaria entre o Ocidente e o Oriente, réuni à Palmela en février 2005. Je tiens à remercier mon amie Isabel Cristina Ferreira Fernandes, organisatrice de la rencontre, de m'avoir donné l'autorisation de le publier également ici.
}

Cahiers de Recherches Médiévales, 15, 2008 
agi dans un cadre avant tout national ${ }^{1}$. Pas plus que ses homologues, le Temple, pourtant étroitement lié à la défense de la Syrie franque jusqu'à sa disparition tragique, n'échappe aujourd'hui à un tel regard. Dans les royaumes de Castille, de León et de Portugal, entre lesquels se partageait la province la plus occidentale de l'ordre ${ }^{2}$, qui, à partir du milieu du XIII ${ }^{\mathrm{e}}$ siècle, ne fut plus nécessairement située sous l'autorité d'un responsable unique ${ }^{3}$, les liens des Templiers locaux avec l'Orient latin sont en effet pratiquement ignorés. Dans la synthèse de Gonzalo Martínez Díez consacrée à l'institution dans l'ensemble castillano-léonais, qui, publiée en 1993, fait aujourd'hui encore figure de référence, la Terre sainte est à peine mentionnée ${ }^{4}$. Elle ne l'est pas davantage dans le récent travail de doctorat de José Valente qui, centré sur le Portugal, en vient à défendre une «nationalisation» du Temple, poussant à l'extrême la perspective d'autonomie à l'égard de l'Orient que Carlos de Ayala Martínez, depuis dix ans bientôt, a proposé d'adopter pour écrire l'histoire de l'ordre à l'échelle des royaumes hispaniques occidentaux ${ }^{6}$.

Majoritaire dans la péninsule Ibérique, un tel point de vue historiographique n'est pas sans poser problème. Faut-il admettre que la défense de la Syrie franque,

\footnotetext{
${ }^{1} \mathrm{Ph}$. Josserand, Église et pouvoir dans la péninsule Ibérique. Les ordres militaires dans le royaume de Castille (1252-1369), Madrid, Casa de Velázquez, 2004, p. 583-647.

${ }^{2}$ Henri de Curzon (éd.), La règle du Temple, Paris, 1886, p. 80, art. 87.

${ }^{3}$ M. Barber, The New Knighthood. History of the Order of the Temple, Cambridge, 1994, p. 380, n. 80, C. de Ayala Martínez, «Frontera castellano-leonesa y órdenes militares. Problemas de jurisdicción (siglos XII-XIII) », Actas de las II Jornadas hispano-portuguesas de historia medieval, éd. V. Á. Álvarez Palenzuela, Madrid, 1999, p. 66-67.

${ }^{4}$ G. Martínez Díez, Los Templarios en la Corona de Castilla, Burgos, Editorial La Olmeda, 1993, p. 44 et 53.

${ }^{5}$ J. Valente, « Soldiers and Settlers. The Knights Templar in Portugal, 1128-1319 », Thèse de doctorat, Santa Barbara, University of California, 2002, p. 117 et p. 120-121: «There is therefore little, if any, of a transnational mentality in the Portuguese Templars in the sense that they could not be dissociated from the interests of the Portuguese kings because the grants made to the order were made not to a branch of an international order, but to those Templars in regno ».

${ }^{6}$ C. de Ayala Martínez, «Las órdenes militares y los procesos de afirmación monárquica en Castilla y Portugal (1250-1350)», As relações de fronteira no século de Alcanices. IV Jornadas luso-espanholas de história medieval, éd., L. A. da Fonseca, Porto, 1998 (2 vol.), t. II, p. 1293, et «Frontera castellano-leonesa y órdenes militares. Problemas de jurisdicción (siglos XII-XIII) », Jornadas de cultura hispano-portuguesa, éd. V. Á. Álvarez Palenzuela, Madrid, 1999, p. 67. Récemment l'auteur a radicalisé sa position en faisant valoir qu' «una regionalización administrativa que obedezca a criterios de imposición política por parte de la monarquía hispánica más poderosa, y que además se consolide sobre la base de un ámplio ámbito peninsular, apunta hacia una autonomía mayor en el seno de la estructura de la orden, una autonomía respecto a ella, inversamente proporcional a la mayor dependencia respecto al monarca favorecedor del nuevo esquema, en este caso Fernando III » (Id., « Fernando III y las órdenes militares », Fernando III y su tiempo [1201-1252], Congreso de estudios medievales, Ávila, 2003, p. 83).
} 
qui, à partir du milieu du XIII ${ }^{\mathrm{e}}$ siècle, reposait largement sur les ordres militaires ${ }^{7}$, a été pour les Templiers castillans et portugais, à la différence de leurs coreligionnaires catalans et aragonais ${ }^{8}$, une préoccupation secondaire? Doit-on penser que l'Outre-mer, dont Alain Demurger a récemment rappelé qu'il était le but suprême de l'engagement dans l'ordre du Temple', constituait pour les frères originaires de l'Occident ibérique un simple horizon théorique? Les actes du procès sur lesquels s'est fondé l'historien français pour montrer combien la Terre sainte restait au début du XIV ${ }^{\mathrm{e}}$ siècle consubstantielle au projet templier invitent à ne pas répondre trop rapidement par l'affirmative à ces deux questions. Dans le royaume de Castille pour lequel, à la différence du Portugal ${ }^{10}$, d'importants vestiges de l'enquête conduite en 1310 sont conservés ${ }^{11}$, la plupart des frères interrogés affirmèrent s'être engagés au moment de leur profession à venir en aide à l'Orient latin de tout leur pouvoir. Et succurere Terre sancte pro posse : à travers ces mots ou sous une forme équivalente qui, en plusieurs cas, peut même faire référence à Jérusalem, l'ensemble des chevaliers du Temple dont on possède la déposition dans les actes du procès de Medina del Campo et près des deux tiers des sergents interrogés à leur suite ont manifesté un désir de se mobiliser pour l'Orient latin'12. Les fragments de l'enquête

\footnotetext{
${ }^{7}$ Le fait a été mis en avant par M. Barber, «Supplying the Crusader States: the Role of the Templars ", The Horns of Hattin, éd. B. Kedar, Jérusalem, 1992, p. 314-317, repris dans Id., Crusaders and Heretics (Thirteenth to Fourteenth Centuries), Aldershot, Ashgate, 1995, XII, A. Demurger, Vie et mort de l'ordre du Temple, Paris, Éditions du Seuil , 1989², p. 231, et P.V. Claverie, «L'ordre du Temple au cœur d'une crise politique majeure : la Querela Cypri des années 1279-1285 », Le Moyen Âge, 104, 1998, p. 496.

${ }^{8}$ Pour la Couronne d'Aragon, les liens des Templiers avec l'Orient ont été soulignés par A. Forey, The Templars in the Corona de Aragón, Londres, 1973, p. 323-332, et J. M. Sans i Travé, Els Templers catalans. De la rosa a la creu, Lérida, Pagès, 1996, p. 222-225 et p. 227228, et, pour le cas particulier de Tortosa, par L. Pagarolas i Sabaté, Els Templers de les terres de l'Ebre (Tortosa). De Jaume I fins a l'abolició de l'Orde (1213-1312), Tarragone, Diputació de Tarragona, 1999 (2 vol.), t. I, p. 110 et p. 279-280.

${ }^{9}$ A. Demurger, « Outre-mer. Les passages des Templiers en Orient d'après les dépositions du procès », Chemins d'outre-mer. Études sur la Méditerranée médiévale offertes à Michel Balard, éd. D. Coulon, C. Otten-Froux, P. Pagès et D. Valérian, Paris, Publications de la Sorbonne, 2004 (2 vol.), t. I, p. 218.

${ }^{10}$ Pour le Portugal, on ne conserve pas d'interrogatoires du procès, contrairement à l'opinion de M. Barber, The Trial of the Templars, Cambridge, 1978, p. 213, trad. fr. Le procès des Templiers, Rennes, Presses Universitaires, 2002, p. 237, et d'A. Demurger, Les Templiers. Une chevalerie chrétienne au Moyen Âge, Paris, Éditions du Seuil, 2005, p. 458, qui lient à ce royaume l'enquête conduite dans la ville galicienne d'Orense sous l'autorité de l'évêque de Lisbonne.

${ }^{11} \mathrm{Ph}$. Josserand, Église et pouvoir dans la péninsule Ibérique. Les ordres militaires dans le royaume de Castille (1252-1369), Madrid, Casa de Velázquez, 2004, p. 53-54, n. 108.

${ }^{12}$ Conservé à l'Archivio Segreto Vaticano dans le fonds Castel Sant'Angelo, le document rapportant l'interrogatoire, en dépit d'importantes lacunes, a été publié par J. M. Sans i Travé, «L'inedito processo dei Templari in Castiglia (Medina del Campo, 27 aprile 1310)», Acri 1291. La fine della presenza degli ordini militari a Terra Santa e i nuovi orientamenti del secolo XIV, éd. F. Tommasi, Pérouse, 1996, p. 249-264.
} 
conduite à Orense, bien qu'infiniment moins explicites, ne semblent en aucune façon démentir ce choix ${ }^{13}$.

Confrontés comme tous leurs coreligionnaires en Occident à la nécessité de s'investir dans une mesure croissante à partir du milieu du $\mathrm{XIII}^{\mathrm{e}}$ siècle pour défendre la Terre sainte, les Templiers castillans, à croire le témoignage qu'ils ont donné au procès, ne paraissent pas s'être dérobés. Leur engagement en Syrie puis, après 1291, à Chypre reste toutefois entièrement à étudier. Plus modeste que celui des frères des royaumes de France et d'Aragon, du fait de ressources davantage limitées ${ }^{14}$, il n'en dépasse pas moins la pauvre image offerte par l'historiographie ${ }^{15}$, qui, à trop raisonner dans un cadre national, est incapable de prendre en compte un ensemble documentaire particulièrement éclaté, dont la presque totalité échappe aux dépôts castillans $^{16}$. De 1'Arquivo Nacional da Torre do Tombo à l'Arxiu de la Corona d'Aragó, des Archives Nationales de France à l'Archivio Segreto Vaticano ou des Patent Rolls du Public Record Office de Londres à la Bibljoteca Nazzjonali de La Valette, l'enquête, qu'il convient d'étendre aux sources chronistiques produites en Orient aux XIII ${ }^{\mathrm{e}}$ et $\mathrm{XIV}^{\mathrm{e}}$ siècles, défie toute prétention d'exhaustivité. Aux pages qui suivent on pourra donc à coup sûr faire grief de méconnaître bien des éléments éclairant l'investissement des Templiers castillans en Terre sainte, mais peut-être y trouvera-t-on aussi la preuve, si vraiment il en fallait une, qu'un tel phénomène mérite que l'historiographie se mobilise pour l'analyser plutôt que de lui opposer, selon les cas, le déni ou le silence.

Bien que les études qui traitent du Temple en Castille n'en disent rien, certains frères de la province ont combattu dans l'Orient méditerranéen. Quelquesuns parmi eux firent même outre-mer d'assez longs séjours. Les traces documentaires de leur engagement sont minces sans doute, mais la recherche reste à conduire et ses résultats pourraient surprendre, comme l'illustre l'exemple de frère

\footnotetext{
${ }^{13}$ C. G. von Murr, « Anhänge zur Geschichte der Tempelherren », dans Id., Über den wahren Ursprung der Rosenkreuzer und des Freymaurerordens, Sulzbach, 1803, p. 146.

${ }^{14}$ M. Barber, The New Knighthood. History of the Order of the Temple, Cambridge, 1978, p. 246, et A. Forey, The Fall of the Templars in the Crown of Aragon, Aldershot, Ashgate, 2001, p. 164.

${ }^{15}$ Dans l'actuelle méconnaissance des relations entre les Templiers castillans et l'Orient, A. Demurger, Les Templiers. Une chevalerie chrétienne au Moyen Âge, Paris, Éditions du Seuil, 2005 , p. 248, a récemment rappelé que les sources n'étaient pas seules responsables, bien que leur pauvreté soit indéniable par rapport à celles dont on dispose pour la Couronne d'Aragon. ${ }^{16}$ Par deux fois déjà, je me suis penché sur ce dossier: Ph. Josserand, "In servitio Dei et domini regis. Les ordres militaires du royaume de Castille et la défense de la Chrétienté latine : frontière et enjeux de pouvoir $\left(\mathrm{XII}^{\mathrm{e}}-\mathrm{XIV}^{\mathrm{e}}\right.$ siècles) », Identidad y representación de la frontera en la España medieval (siglos XI-XIV). Actas del seminario de la Casa de Velázquez $y$ de la Universidad Autónoma de Madrid (14-15 de diciembre de 1998), éd. C. de Ayala Martínez, P. Buresi et Ph. Josserand, Madrid, 2001, p. 89-111, notamment p. 92-96, et «Entre Orient et Occident : l'ordre du Temple dans le contexte castillan du règne d'Alphonse $\mathrm{X}$ », Alcanate. Revista de Estudios Alfonsíes, 2, 2000-2001, p. 131-150, en particulier p. 133-140.
} 
Reddecœur, ce chevalier de l'ordre célèbre pour être intervenu dans les démêlés politiques qui ont agité les dernières années d'existence du comté de Tripoli, auquel il convient que la critique restitue son origine asturienne.

Désigné sous le nom qui lui est resté attaché jusqu'à aujourd'hui par le Templier de Tyr, ce chroniqueur anonyme, en réalité issu de la petite noblesse d'Orient, dont il dut partager le quotidien à partir de 1285 dans l'entourage de Guillaume de Beaujeu ${ }^{17}$, Reddecœur apparaît étroitement mêlé à la crise qui, peu après l'avènement de Bohémond VII, opposa le prince d'Antioche au seigneur de Gibelet pour le contrôle de Tripoli ${ }^{18}$. Déclenché en 1278, pour autant que la chronologie permette de l'établir' ${ }^{19}$, ce conflit, dans lequel le Temple a d'emblée pris parti pour Guy de Gibelet ${ }^{20}$, rebondit en 1282 quand, après une trêve précaire, celuici tenta par la mer de se rendre maitre de Tripoli avec la complicité de Reddecœur, le commandeur de la place ${ }^{21}$. Faute de coordination, le coup de main fit long feu et, contraint de se réfugier dans la maison du Temple, dont les membres s'étaient portés à sa rencontre, Guy de Gibelet n'eut d'autre choix que de se soumettre à Bohémond d'Antioche, qui, jouant de sa parole, le condamna à périr emmuré dans une fosse du château de Néphin ${ }^{22}$; l'infortuné seigneur fut même contraint à des aveux dont le

17 G. Airaldi, "'Je suis Bertrand de Gibelet'», Chemins d'outre-mer. Études sur la Méditerranée médiévale offertes à Michel Balard, éd. D. Coulon, C. Otten-Froux, P. Pagès et D. Valérian, Paris, Publications de la Sorbonne, 2004 (2 vol.), t. I, p. 25, sur la foi de l'édition critique récente Cronaca del Templare di Tiro (1243-1314), éd. L. Minervini, Naples, Liguori Editore, 2000.

${ }^{18}$ Synthétisée par J. Richard, «Les comtes de Tripoli et leurs vassaux sous la dynastie antiochénienne ", Crusade and Settlement. Papers read at the $1^{\text {st }}$ Conference of the Society for the study of the crusades and the Latin East and presented to R. C. Smail, éd. P. Edbury, Cardiff, 1985, p. 223-224, repris dans Id., Croisades et États latins d'Orient, Aldershot, Ashgate, 1992, XI, cette crise complexe a été envisagée sous l'angle du Temple par H. Nicholson, The Knights Templar. A New History, Stroud, 2001, p. 84, et A. Demurger, Les Templiers. Une chevalerie chrétienne au Moyen Âge, Paris, Éditions du Seuil, 2005, p. $362-$ 363.

${ }^{19}$ La difficulté de dater avec exactitude les événements de la crise a été signalée par P.-V. Claverie, «L'ordre du Temple au cour d'une querelle politique majeure : la Querela Cypri des années 1279-1285 », Le Moyen Âge, 104, 1998, p. 498-499, n. 12.

${ }^{20}$ Cronaca del Templare di Tiro (1243-1314), éd. L. Minervini, Naples, Liguori Editore, 2000, p. 144-146, «Et le seignor de Giblet ala a Acre, et se fist confrere dou Temple, et eust grant amisté au maistre frere Guillaume de Biaujeu, quy ly promist de luy aider de quant que il porra $»$.

${ }^{21}$ Ibid., p. 156, «Et vint a Triple et ariva entre la mayson dou Temple et les freres Prechours, et desendy entre luy et sa gent et les chevaus, et puis fist esfondrer la guallee et la grant saitie et laissa la petite entiere, et s'en ala au Temple, et demanda le coumandour, frere Renddecuer, et il n'y estoit pas, dont il fu moult mescoragés ».

${ }^{22}$ Ibid., p. 156-158, «Et le seignor de Gibelet et ses .ii. freres et lor couzin Guillaume de Gibelet et Andrey de Clapiere, le prince les manda a Nefin et les fist metre en une foce et masouner et cloreles dedans, et morurent de faim $»$. 
texte est conservé23, à travers lesquels il se présente comme un simple jouet des Templiers et de leur maître Guillaume de Beaujeu ${ }^{24}$. C'est auprès de ce dernier une fois encore que, quelques années plus tard, Reddecœur apparaît dans les deux ultimes mentions que l'on conserve de lui, envoyé depuis Acre pour prévenir les habitants de Tripoli d'une attaque mamelouke ${ }^{25}$, lors de laquelle, le 26 avril 1289, il fut capturé tandis que la cité tombait au pouvoir des musulmans ${ }^{26}$.

Présent en Orient depuis huit ans au moins lorsqu'il fut fait prisonnier ${ }^{27}$, Reddecœur n'est au mieux qu'un nom dans les histoires générales de la croisade et du Temple ${ }^{28}$. Pourtant, il existe la matière pour préciser son identité. Récemment, Alain Demurger a observé qu'il était « un chevalier important de l'ordre qui avait été commandeur de Tripoli $»^{29}$. Reprenant les données rapides fournies par le Templier de Tyr, qui attribuait au frère " grant aparance », l'historien français a néanmoins omis de relever l'unique information chronistique dont on puisse se prévaloir en terme d'origine puisque, selon son contemporain, Reddecœur était un «frère chevalier espaignol $»^{30}$. Occultée par la francisation de son nom dans le récit, où

${ }^{23}$ Conservé aux Archives Nationales de France, ce document a été publié par L. de MasLatrie, Histoire de l'île de Chypre, Paris, 1852-1861 (4 vol.), t. III, p. 662-668. Daté par son éditeur du 26 février 1282, il est en réalité postérieur d'un an, comme l'ont fait valoir J. Richard, "Les comtes de Tripoli et leurs vassaux sous la dynastie antiochénienne ", Crusade and Settlement, éd. P. Edbury, Cardiff, 1985, p. 223-224, n. 44, et P.-V. Claverie, «L'ordre du Temple au cœur d'une querelle politique majeure : la Querela Cypri des années 1279-1285 ", Le Moyen Âge, 104, 1998, p. 502, n. 28, attentifs à prendre en compte la particularité du style chypriote, qui commence au $1^{\text {er }}$ mars.

${ }^{24}$ A. Demurger, Jacques de Molay. Le crépuscule des Templiers, Paris, Payot, $2007^{2}$, p. 71.

${ }^{25}$ Cronaca del Templare di Tiro (1243-1314), éd. L. Minervini, Naples, Liguori Editore, 2000, p. 193.

${ }^{26}$ Ibid., p. 198 : «Et mourut en la bataille frere Piere de Moncade, coumandour dou Temple, et Guilerme de Cardone, frere dou Temple, et fu pris vif frere Reddecuer, et frere Huguet, fis dou conte d'Enpures, frere dou Temple, et meÿmes furent mort et pris aucuns freres de l'Ospitau de Saint Johan ».

${ }^{27}$ Une telle durée est minimale puisqu'en 1281, Reddecœur, qui était commandeur de Tripoli, à en croire le témoignage au procès du frère sergent Tomás de Pamplona, qu'il avait reçu dans l'ordre (J. Michelet, Le procès des Templiers, Paris, Éditions du C.T.H.S., 1987 [2 vol.], t. II, p. 16), détenait l'une des plus importantes dignités de la milice, ce qui, comme l'ont signalé A. Forey, The Templars in the Corona de Aragón, Londres, 1973, p. 315, et J. M. Sans i Travé, Els Templers catalans. De la rosa a la creu, Lérida, Pagès, 1996, p. 197-198, reprenant La règle du Temple, éd. H. de Curzon, Paris, 1886, p. xix, impliquait évidemment qu'il eût déjà acquis certaine renommée.

${ }^{28}$ R. Grousset, Histoire des croisades et du royaume franc de Jérusalem, Paris, 1936, rééd. Paris, Perrin, 1991 (3 vol.), t. III, p. 690, S. Runciman, A History of the Crusades, Cambridge, 1951, trad. fr. Histoire des Croisades, Paris, Dagorno, 1998, p. 980, ou M. Barber, The New Knighthood. A History of the Order of the Temple, Cambridge, 1994, p. 174 et 176.

${ }^{29}$ A. Demurger, Jacques de Molay. Le crépuscule des Templiers, Paris, Payot, $2007^{2}$, p. 78.

${ }^{30}$ Cronaca del Templare di Tiro (1243-1314), éd. L. Minervini, Naples, Liguori Editore, 2000, p. 194 : «Et quant le soudan fu venu plus avant, le maistre manda i. autre mesage de grant aparance, si fu frere Reddeceur, frere chevalier espaignol, et lor fist saver coument le 
alternent les graphies Renddecuer, Reddeceur et Reddecuer ${ }^{31}$, l'origine hispanique du dignitaire ressort de l'acte enregistrant les aveux de Guy de Gibelet, qui, chaque fois qu'il se réfère à lui, recourt à la forme Ruidecuer ${ }^{32}$. Jouant avec finesse de l'outil anthroponymique dont on ne saurait trop souligner les potentialités pour l'étude des ordres militaires ${ }^{33}$, Pierre-Vincent Claverie a le premier restitué au frère sa véritable identité : proposant de le nommer Rui de Cuero, il a très justement placé l'accent sur son origine castillane ${ }^{34}$. À cette appellation de Cuero, dont, certes, il existe un équivalent toponymique ${ }^{35}$, il est possible qu'il faille préférer Cuera ou Cueras, qui sont l'une et l'autre aussi des formes attestées dans les Asturies ${ }^{36}$. La discussion est simplement de détail, et l'essentiel tient bel et bien à l'identité castillane du commandeur de Tripoli auquel la critique, sur la base du récit du Templier de Tyr, a prêté le nom francisé de Reddecœur.

Ignoré de l'historiographie espagnole, ce dignitaire lié au maître Guillaume de Beaujeu, au conseil duquel il a même pu participer, ne devait pas être au début des années 1280 en Orient le seul Templier provenant des régions occidentales de la péninsule Ibérique. À croire la déposition de Guy de Gibelet enregistrée au château de Néphin, alors qu'il était aux mains de Bohémond d'Antioche, plusieurs frères de l'entourage de Reddecœur, qui ont eux aussi trempé dans l'action dirigée contre Tripoli, pourraient partager la même origine géographique que celui-ci. Revenant sur les débuts de la malheureuse entreprise qui devait valoir une mort terrible à son principal promoteur, le document rapporte l'implication d'un Templier appelé Ferrant, présenté comme le compagnon de Reddecœur au sein de la commanderie

soudan [venait], et a tout se furent il entre .ii. dou creire ou non, et toute fois ordenerent leur fait, et Reddeceur revint a Acre, et le soudan vint devant Triple ».

${ }^{31}$ Ibid., p. 156 (Renddecuer), p. 194 (Reddeceur deux fois) et p. 198 (Reddecuer).

${ }^{32}$ L. de Mas-Latrie, Histoire de l'île de Chypre, Paris, 1852-1861 (4 vol.), t. III, p. 665-666. De manière comparable, lorsqu'il s'est référé au dignitaire qui l'avait fait templier, le frère sergent Tomás de Pamplona, au moment du procès, a évoqué «fratrem quondam Rodoricum de Cuyre militem, tunc preceptorem Tripolitanum » (J. Michelet, Le procès des Templiers, Paris, Éditions du C.T.H.S., 1987 [2 vol.], t. II, p. 16). Ces deux versions ne sont éloignées qu'en apparence, puisque, dans le castillan de l'époque, Ruy et Rodrigo étaient équivalents.

${ }^{33} \mathrm{Ph}$. Josserand, Église et pouvoir dans la péninsule Ibérique. Les ordres militaires dans le royaume de Castille (1252-1369), Madrid, Casa de Velázquez, 2004, p. 383-384.

${ }^{34}$ P.-V. Claverie, «Le statut des Templiers capturés en Orient durant les croisades », La liberazione dei captivi tra Cristianità e Islam. Oltre la crociata e il gihad: tolleranza e servizio umanitario, éd. G. Cipollone, Cité du Vatican, 2000, p. 504, et, Id., L'ordre du Temple en Terre sainte et à Chypre au XIII ${ }^{e}$ siècle, Nicosie, 2005 (3 vol.), t. I, p. 209, et t. II, p. 338.

${ }^{35} \mathrm{Au}$ nord-ouest d'Oviedo, dans la vallée du Nalón, il existe, à proximité de la petite ville de Grado, un village qui porte le nom de Cuero, dont le dignitaire du Temple pourrait être originaire.

${ }^{36}$ À l'extrémité orientale des Asturies, la Sierra de Cuera, dans l'arrière-pays de Llanes, se dresse entre le littoral et le massif des Picos de Europa, dont elle est séparée par l'étroite vallée de Cabrales. À l'opposé de la province, on trouve à quelques kilomètres au sud-ouest de Cangas del Narcea un petit village du nom de Cueras. 
tripolitaine de l'ordre ${ }^{37}$. Précédemment déjà, un membre de cette même maison, le prêtre Johan Arobe, serait entré en contact avec Guy de Gibelet, du moins aux dires de ce dernier ${ }^{38}$, tentant par deux fois, sans plus de succès, de lui livrer le contrôle de la ville ${ }^{39}$. À travers de tels noms, à peine francisés par un notaire oriental de culture d'oïl ${ }^{40}$, il n'est pas difficile d'identifier deux frères originaires de l'Occident péninsulaire, sans doute Fernán et Juan Arroba, pour lesquels, à défaut de tout autre élément d'information, on ne saurait pourtant affirmer avec autant de certitude que dans le cas de Reddecœur qu'ils sont de naissance castillane plutôt que portugaise. Si un doute subsiste sur ce point, la confession de Guy de Gibelet n'en révèle pas moins que des Templiers issus des terres ibériques occidentales ont, plus qu'on le croit, servi en Orient ${ }^{41}$.

Pour évaluer un tel mouvement, l'anthroponymie est sans doute un marqueur aussi fragile qu'insuffisant. On ne saurait à l'évidence s'en tenir à elle, mais on aurait tort tout autant de mépriser ses enseignements car l'engagement de Reddecœur et de ses compagnons qui s'illustrèrent dans les dernières années de la domination latine à Tripoli participe d'un investissement castillan en Orient beaucoup plus profond que ne le prétend l'historiographie. Au-delà du cas particulier emblématique auquel je me suis jusqu'ici attaché, c'est le phénomène

\footnotetext{
${ }^{37}$ L. de Mas-Latrie, Histoire de l'île de Chypre, Paris, 1852-1861 (4 vol.), t. III, p. 665 : « De quoi le maistre fu durement esmeu, et manda letres de creance par frere Ferrant, compaignon de frere Ruidecuer, comandor de Triple, au sire de Gibelet, esqueles se conteneit qu'il deust creire et faire sans faille ce que sire Pol li direit, et que s'il nel' faisait, il n'aureit plus ne s'aye ne sa maintenance $»$.

${ }^{38}$ Ibid., p. 664 : «Un frere du Temple qui a nom frere Stiene, et le susnomé prestre, Johan Arobe, du Temple, manderent letres au dit sire de Gibelet, et li firent à savoir que le prince estoit durement ataint, et qu'il s'en venist hastivement à Triple, car tot la gent de la vile le voloit, et qu'il troveroit à la maison dou Temple et à la rive gent à cheval et à pié assés qui l'atendroient, et qu'il venist seurement ».

${ }^{39}$ Sans faire mention du frère prêtre du Temple, J. Richard, «Les comtes de Tripoli et leurs vassaux sous la dynastie antiochénienne », Crusade and Settlement, éd., P. Edbury, Cardiff, 1985, p. 219, et A. Demurger, Jacques de Molay. Le crépuscule des Templiers, Paris, Payot, $2007^{2}$, p. 72, ont chacun rapporté l'échec des deux premières attaques lancées contre Tripoli après la paix précaire négociée en 1279 .

${ }^{40}$ L. de Mas-Latrie, Histoire de l'île de Chypre, Paris, 1852-1861 (4 vol.), t. III, p. 668.

${ }^{41}$ Aux côtés de Reddecœur, on sait grâce à la déposition du frère sergent Tomás de Pamplona (J. Michelet, Le procès des Templiers, Paris, Éditions du C.T.H.S., 1987 [2 vol.], t. II, p. 16) qu'en 1281 servait à Tripoli un certain Rodrigo Yáñez, que l'on doit sans doute identifier à celui qui fut le dernier maitre provincial du Temple en Castille. Si l'on peut penser comme le souligne P.-V. Claverie, L'ordre du Temple en Terre sainte et à Chypre au XIII siècle, Nicosie, 2005 (3 vol.), t. II, p. 209-210, que ce frère « retira manifestement un grand bénéfice de son séjour en Terre sainte ", on ne saurait en revanche dater avec l'auteur sa désignation à la tête de la province castillane de l'ordre vers 1290, puisqu'elle fut en réalité postérieure de douze ans (Ph. Josserand, « Rodrigo Yáñez », Dictionnaire européen des ordres militaires au Moyen Âge, éd. Ph. Josserand et N. Bériou, Paris, Fayard, 2008, à paraître).
} 
dans son ensemble qu'il convient désormais d'étudier en croisant le plus possible des sources extrêmement dispersées.

Dépendant toujours davantage de l'Occident au fur et à mesure que la pression musulmane se développait, les États croisés de Terre sainte ont supposé pour leur défense d'organiser un réseau d'approvisionnement très large ${ }^{42}$, dont les historiens qui l'ont étudié ont souligné combien il a laissé peu de traces documentaires ${ }^{43}$. Fortement intéressé à ces échanges d'hommes, de vivres et d'argent, le Temple ne fait pas exception à la norme et, à défaut d'archives centrales

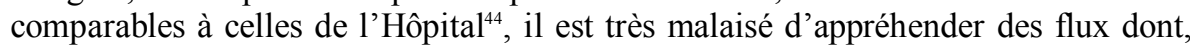
dans les provinces ibériques occidentales de l'ordre, la critique a excipé du peu de lisibilité pour nier la réalitéts.

Bien que l'historiographie les présente comme marginales ${ }^{46}$, les interventions des autorités centrales du Temple en terre castillane ont tendu dès le deuxième quart du XIII ${ }^{e}$ siècle à se renforcer sous l'effet de besoins croissants, que plusieurs frères, comme plus tard le maître provençal Pons de Brozet, ont pu être chargés de relayer ${ }^{47}$. Déjà en 1223 , la force du contrôle magistral ressortait de la requête pontificale adressée au supérieur de l'ordre afin qu'il ne fît pas grief au dignitaire provincial d'avoir souscrit de lourds emprunts pour répondre aux nécessités de la Reconquête ${ }^{48}$. Vingt ans plus tard, à la veille de la bataille de La Forbie, ce fut en Orient qu'à

${ }^{42}$ M. Barber, "Supplying the Crusader States: the Role of the Templars », The Horns of Hattin, éd. B. Kedar, Jérusalem, 1992, p. 317, repris dans Id., Crusaders and Heretics (Thirteenth to Fourteenth Centuries), Aldershot, 1995, XII. Récemment, ce système a fait l'objet d'une approche intéressante de la part de J. Bronstein, The Hospitallers and the Holy Land. Financing the Latin East, 1187-1274, Woodbridge, The Boydell Press, 2005.

${ }^{43}$ A. Forey, "Cyprus as a Base for Crusading Expeditions to the West ", Cyprus and the Crusades, éd. N. Coureas et J. Riley-Smith, Nicosie, 1995, p. 75 : « Much of the evidence about the supplying of crusading forces from a distance in the thirteenth century is imprecise ».

${ }^{44}$ R. Hiestand, «Zum Problem des Templerzentralarchivs », Archivalische Zeitschrift, 76, 1980, p. 17-37.

${ }^{45}$ Un manque de sources tout à fait comparable peut être observé pour des espaces dont nul ne discute pourtant les liens avec l'Orient latin, à l'exemple du Languedoc et de la Provence, étudiés par D. Selwood, Knights of the Cloister. Templars and Hospitallers in CentralSouthern Occitania (c. 1100 -c. 1300), Woodbridge, The Boydell Press, 1999, p. 174, et D. Carraz, L'ordre du Temple dans la basse vallée du Rhône, 1124-1312. Ordres militaires, croisades et sociétés méridionales, Lyon, Presses Universitaires, 2005, p. 282-283.

${ }^{46}$ G. Martínez Díez, Los Templarios de la Corona de Castilla, Burgos, 1993, p. 61-62, et C. de Ayala Martínez, «Fernando III y las órdenes militares », Fernando III y su tiempo [120112527. Congreso de estudios medievales, Ávila, 2003, p. 83-84.

${ }^{47} \mathrm{~J}$. Gay, Les registres de Nicolas III (1277-1280). Recueil des bulles de ce pape publiées ou analysées d'après les manuscrits originaux des Archives du Vatican, Paris, 1898, p. 307, doc. 676 et 677.

${ }^{48}$ D. Mansilla, La documentación pontificia de Honorio III (1216-1227), Rome, 1965, p. 324325, doc. 447. 
l'initiative des plus hautes autorités templières a été vidée la querelle qui, depuis la disparition de Guillaume Fouque, opposait les frères castillans et portugais, tenant pour un successeur différent ${ }^{49}$. Dans l'Occident hispanique, le contrôle du maître n'a toutefois pas occasionné, à la différence de l'Aragon ${ }^{50}$, d'intervention directe ${ }^{51}$. Pour autant, on ne saurait en déduire qu'il fut plus lâche. Désireux d'impliquer la Castille dans la défense de l'Orient ${ }^{52}$, Jacques de Molay a tenté lors d'une vaste tournée, ponctuée en 1296 par la réunion du chapitre général en $A_{r l e s}{ }^{53}$, de s'assurer le concours des frères de la province, favorisant leur ancien maître, le Portugais João Fernandes, passé au service du pape $^{54}$. Jouant de méthodes déjà éprouvées par Thomas Bérard et Guillaume de Beaujeu, qui avaient imposé leur accord à diverses

\footnotetext{
49 Brièvement évoqué par J. Valente, «Soldiers and Settlers. The Knights Templar in Portugal, 1128-1319», Thèse de doctorat, Santa Barbara, University of California, 2002, p. 186, le fait ressort d'un « retrait » de l'ordre du Temple inséré dans La règle du Temple, éd. H. de Curzon , Paris, 1886, p. 302-304, art. 582-583 : «Et le maistre manda comandeor en Espaine et manda a ces .ii. freres qu'i venissent en cest païs, et il vindrent et crierent merci de ceste chose devant le maistre et le couvent. Et li maistres et li couvent virent que le dui frere avoient perdue la maison et le mistrent en respit por ce que il estoient dui prodome et de bone vie et de bone religion, et que la chose estoit novele [...] Mais sachiés qu'il avoient perdue la maison selonc nos establissemenz por ce que il avoient usé de pooir dont il ne devoient user ». ${ }^{50}$ Pour cette province, en 1275 et en 1294, des visites de Guillaume de Beaujeu et de Jacques de Molay ont été relevées par A. Forey, The Templars in the Corona de Aragón, Londres, 1973, p. 329, et A. Demurger, Jacques de Molay. Le crépuscule des Templiers, Paris, Payot, 2007², p. 118-121. Attestée par un acte de l'Arxiu de la Corona d'Aragó publié par L. Pagarolas i Sabaté, Els Templers de les terres de l'Ebre (Tortosa). De Jaume I fins a l'abolició de l'Orde (1213-1312), Tarragone, Diputació de Tarragona, 1999 (2 vol.), t. II, p. 197-198, doc. 171, cette ultime intervention aurait dû avoir lieu l'année précédente (A. Forey, "Letters of the Last Two Templar Masters », Nottingham Medieval Studies, 44, 2001, p. 156).

${ }^{51}$ Mis en avant par A. Forey, The Templars in the Corona de Aragón, Londres, 1973, p. 343 , n. 184, et A. Demurger, Jacques de Molay. Le crépuscule des Templiers, Paris, Payot, 2007², p. 214, un projet de visite a simplement été conçu en 1307, ainsi qu'il ressort d'une lettre de Pere de Sant-Just adressée à Jacques de Molay, publiée par H. Finke, Papsttum und Untergang des Templerordens, Münster, 1907 (2 vol.), t. II, p. 37, doc. 24.

${ }^{52}$ S. Schein, Fideles Crucis. The Papacy, the West and the Recovery of the Holy Land (12741314), Oxford, 1991, p. 201.

${ }^{53}$ A. Demurger, Jacques de Molay. Le crépuscule des Templiers, Paris, Payot, 2007², p. 124 138.

${ }^{54}$ Devenu chambrier du pontife à l'été 1296, João Fernandes a reçu lors du chapitre d'Arles les commanderies de Faro, Canabal, Neira et Ceinos de Campos en vertu d'une lettre de Jacques de Molay, incluse dans une lettre de Boniface VIII. De ce document conservé à l'Archivio Segreto Vaticano, Reg. Vat. 48, f. 143v-144r., dont seule la partie due au pape a bénéficié d'une publication (Les registres de Boniface VIII. Recueil des bulles de ce pape publiées ou analysées d'après les manuscrits originaux des Archives du Vatican, éd. G. Digard, M. Faucond, A. Thomas et R. Fawtier, Paris, 1907-1934 [4 vol.], t. I, col. 547, doc. 1508), je remercie Alain Demurger de m'avoir facilité la copie qu'il s'est procurée.
} 
concessions de franchises ${ }^{55}$, voire réaffirmé leur droit à nommer le supérieur provincial ${ }^{56}$, il favorisa plus que ses devanciers l'action du visiteur général d'Espagne $^{57}$, sur lequel, durant le long mandat de Berenguer de $\mathrm{Cardona}^{58}$, il s'appuya pour mobiliser en Castille, comme dans les autres régions d'Occident, une part croissante des ressources domaniales du Temple ${ }^{59}$.

D'après une bulle de Nicolas IV ${ }^{60}$, les sommes et les produits envoyés par les Templiers en Orient, qualifiés de responsiones, formaient le tiers des rentes de

\footnotetext{
${ }_{55} \mathrm{Au}$ cours du magistère de Thomas Bérard, les chartes de franchise accordées à Castelo Branco, en 1271, et à Valencia del Ventoso, l'année suivante, l'avaient été avec le consentement exprès du couvent d'Outre-mer, d'après les érudits qui au XVIII ${ }^{\mathrm{e}}$ siècle en ont rapporté l'existence (J. A. de Figueiredo, Nova história da militar Ordem de Malta é dos senhores grão-priores della em Portugal, Lisbonne, 1800 [2 vol.], t. II, p. 256, et P. Rodríguez Campomanes, Dissertaciones históricas del orden y cavallería de los Templarios, Madrid, 1747, rééd. Barcelone, 1975, p. 30-31). Cet aval du grand-maître et du couvent se retrouve au temps de Jacques de Molay, par exemple dans un échange arrêté entre le Temple et les Dominicains de Benavente, dont il est fait mention le 14 mai 1298 dans un document publié par R. Fernández Ruiz, Colección diplomática del monasterio de Santo Domingo de Benavente (1228-1390), Benavente, 2000, p. 65, doc. 27.

${ }^{56}$ M. González Jiménez, Diplomatario andaluz de Alfonso X, Séville, 1991, p. 539-540, doc. 509.

${ }^{57}$ Créée au milieu du XIII ${ }^{\mathrm{e}}$ siècle, cette charge, dont le premier titulaire fut le maître castillan Lope Sánchez (J. Miret i Sans, Itinerari de Jaume I el Conqueridor, Barcelone, 1918, p. 385), ne s'est pas immédiatement substituée à celle de visiteur cismarin puisque Francon de Bort est attesté avec ce titre dans l'ouest de la péninsule Ibérique (S. A. Gomes, « A presença das ordens militares na região de Leiria [séculos XII-XV] », As ordens militares em Portugal e no Sul da Europa, éd. I. C. Ferreira Fernandes et P. Pacheco, Lisbonne, 1997, p. 179, doc. 11).

${ }^{58}$ A. Forey, The Templars in the Corona de Aragón, Londres, 1973, p. 329-330 et p. 342, n. 181.

${ }^{59}$ En mai 1302, à peine plus d'un an après être rentré de Chypre, où son lieutenant Pere de Tous avait reçu la délégation des affaires de l'ordre en Castille, Berenguer de Cardona s'est rendu en personne dans la province, désireux, comme il l'a écrit au commandeur de Peñíscola, de remédier à une situation suffisamment grave pour qu'il dût s'en ouvrir à Ferdinand IV, qui s'apprêtait à réunir les Cortes à Medina del Campo : «Fem vos saber [...] divendres 25 dies anatz del mes de maig entram en Medina del Campo e aqui fom molt be e honradament acolliz per lo senyor rey de Castella e per totz los richshomens de la terra. E axi havem parlat ab lo senyor rey de Castella e trobam lo de molt bon enteniment enves [el Temple]. E axi havem ferma esperança que nostres afers vendran acabament donor e a profit del Temple. Encara no haviem tengut capitol [...] mas ja erem de cami per anarnos endeves Çamora per tenir capitol e per endreçar les cases e los logars del Temple, los quals, si a Deu que plau, tornaran en estament de be». Je remercie Alan Forey de m'avoir facilité les transcriptions qu'il a établies de ces deux actes (Arxiu de la Corona d'Aragó, Cancillería real, Cartas reales diplomáticas, Templarios, doc. 181 et 322).
}

${ }^{60}$ A. Forey, The Templars in the Corona de Aragón, Londres, 1973, p. 323. 
chaque commanderie ${ }^{61}$. Théorique, une telle proportion est impossible à vérifier en Castille $^{62}$ et, s'il faut sans doute, comme en Aragon, plutôt la rapprocher du dixième $^{63}$, on ne saurait l'estimer négligeable ${ }^{64}$. En effet, au printemps 1271, le monopole commercial avec l'Outre-mer concédé par Alphonse X à Carthagène et Alicante révèle que le Temple et l'Hôpital étaient alors les plus importants promoteurs des passages à destination de la Terre sainte ${ }^{65}$. Or, au trafic de ces cités, il faut ajouter les flux en partance du sud de la France, en particulier de Marseille ${ }^{66}$ d'où le supérieur castillan du Temple Rodrigo Yáñez partit pour Chypre en $1305^{67}$, et, plus fréquemment sans doute, des ports catalans comme Barcelone ${ }^{68}$, où embarquèrent au printemps 1286 trente chevaux entrés en Aragon à Ariza, pour le convoi desquels Alphonse III donna aux autorités de l'ordre le droit de traverser librement son royaume ${ }^{69}$. À côté des montures dont la Castille semble s'être fait une

${ }^{61}$ Étudié en détail par J. Riley-Smith, The Knights of St John in Jerusalem and Cyprus (c. 1050-1310), Londres, 1967, p. 344-346 et 440-443, ce mécanisme vaut pour tous les ordres militaires internationaux.

${ }^{62}$ Commun à de nombreux espaces, le constat peut être également appliqué à la Provence étudiée par D. Carraz, L'ordre du Temple dans la basse vallée du Rhône, 1124-1312. Ordres militaires, croisades et sociétés méridionales, Lyon, Presses Universitaires, 2005, p. 282-283.

${ }^{63}$ A. Forey, The Templars in the Corona de Aragón, Londres, 1973, p. 323, a fait état de cette proportion pour le début $\mathrm{du} \mathrm{XIV}^{\mathrm{e}}$ siècle sur la base de l'existence d'un frère appelé decimarius ou delmer, préposé à l'organisation des transferts à destination du couvent central de Chypre.

${ }^{64} \mathrm{Ph}$. Josserand, «Entre Orient et Occident : l'ordre du Temple dans le contexte castillan du règne d'Alphonse X », Alcanate. Revista de Estudios Alfonsíes, 2, 2000-2001, p. 135-136.

${ }^{65}$ J. Torres Fontes, Fueros y privilegios de Alfonso X el Sabio al reino de Murcia, Murcie, 1973, p. 111, doc. 100, «Porque es mas açerca et mas endreçado el passage pora ultramar por estos puertos de Alicant et de Cartagenia a todos los omnes de mis reynos et de mio sennorio que allá quisieren passar, tengo por bien et mando que las ordenes del Ospital et del Temple et las otras ordenes et los grandes omnes et los mercaderos de mi tierra que quisieren passar a oltramar, que non fagan el passage por otros lugares sinon por los puertos de Alicant et Cartagenia ».

${ }^{66}$ Relevé par A. Forey, The Templars in the Corona de Aragón, Londres, 1973, p. 326, et M. Barber, " Supplying the Crusader States: the Role of the Templars », The Horns of Hattin, éd. B. Kedar, Jérusalem, 1992, p. 322-323, le rôle essentiel de Marseille pour le Temple en Méditerranée a été récemment étudié par A. Demurger, Les Templiers. Une chevalerie chrétienne au Moyen Âge, Paris, Éditions du Seuil, 2005, p. 340, et D. Carraz, L'ordre du Temple dans la basse vallée du Rhône, 1124-1312. Ordres militaires, croisades et sociétés méridionales, Lyon, Presses Universitaires, 2005, p. 306 et 494-496.

${ }^{67}$ Le passage du dignitaire outre-mer ressort de l'interrogatoire des Templiers à Chypre, publié par K. Schottmüller, Die Untergang des Templerordens, Berlin, 1887 (2 vol.), t. II, p. 208, et traduit en anglais par A. Gilmour-Bryson, The Trial of the Templars in Cyprus. A Complete English Edition, Leyde, 1998, p. 138.

${ }^{68}$ D. Selwood, Knights of the Cloister. Templars and Hospitallers in Southern-Central Occitania (c. 1100 -c. 1300), Woodbridge, The Boydell Press, 1999, p. 191.

${ }^{69}$ Une missive d'Alphonse III d'Aragon à l'officier en charge de l'administration royale à Calatayud rapporte à la date du $1^{\mathrm{er}}$ mars que «propter inopiam equorum quam sustinet Terra 
spécialité $^{70}$, du fait de la renommée attachée aux bêtes élevées sur son sol ${ }^{71}$, ce dernier envoi comprenait des harnais, de la viande séchée et d'autres victuailles aptes à pallier les carences criantes des États croisés d'Orient ${ }^{72}$. Parallèlement à de tels transferts, de l'argent quittait également l'Occident hispanique et, bien que les sources manquent pour le Temple $\mathrm{T}^{73}$, on est fondé à extrapoler la mention du Templier de Tyr qui décrit Guillaume de Beaujeu, sitôt après avoir été promu à la tête de son ordre, parcourant diverses provinces, parmi lesquelles l'Espagne ${ }^{74}$, pour

sancta concessimus magistro Templi quod possit extrahi facere de Castella viginti equos et ipsos eosdem mittere ad partes [trans]marinas et facere eos recolligere in maritimis nostris »; le 27 avril suivant, un ordre du roi à son représentant étend la concession précédente à trente chevaux, dont le transport à partir de la localité d'Ariza est le même jour réglé par un dernier courrier (Arxiu de la Corona d'Aragó, Cancillería real, Reg. 63, fol. 89 ${ }^{\mathrm{r}}$, et Reg. 66 , fol. $57^{\mathrm{v}}$ et $58^{\mathrm{r}}$ ). Pour ces trois documents, je remercie Alan Forey de m'avoir procuré des extraits de ses transcriptions, qui ont utilement complété mes dépouillements.

${ }^{70} \mathrm{Ph}$. Josserand, Église et pouvoir dans la péninsule Ibérique. Les ordres militaires dans le royaume de Castille (1252-1369), Madrid, Casa de Velázquez, 2004, p. 597-598, notamment n. 88. Des mouvements de chevaux en provenance de la péninsule Ibérique vers l'Orient latin témoignent les registres angevins de l'Archivio di Stato de Naples (R. Filangieri, J. Mazzoleni et al., I registri della cancilleria angioina, Naples, 1950-1996 [43 vol.]), qui ont été exploités par A. Demurger, Les Templiers. Une chevalerie chrétienne au Moyen Âge, Paris, Éditions du Seuil, 2005, p. 335, et K. Toomaspoeg, « Le ravitaillement de la Terre sainte. L'exemple des possessions des ordres militaires dans le royaume de Sicile au XIII ${ }^{\mathrm{e}}$ siècle », L'expansion occidentale $\left(X I^{e}-X V^{e}\right.$ siècles) : formes et conséquences. Actes du XXXIII $I^{e}$ Congrès de la Société des historiens médiévistes de l'enseignement supérieur public, Paris, Publications de la Sorbonne, 2003, p. 151. Je remercie ce dernier auteur de m'avoir signalé combien les traces de tels échanges, qui s'appuyaient pour une bonne part sur Messine, sont aujourd'hui rares, faute de registres portuaires pour le Midi italien, bien qu'il soit permis d'estimer qu'ils correspondaient à une pratique assez courante, comme l'a suggéré N. Housley, «I registri angioini ricostruiti e le crociate », Per la storia del Mezzogiorno medievale e moderno. Studi in memoria di Jole Mazzoleni, Naples, 1998 (2 vol.), t. I, p. 146-147.

${ }^{71}$ La réputation d'excellence des chevaux castillans a été mise en avant par Y. Renouard, «Un sujet de recherches : l'exportation de chevaux de la péninsule Ibérique en France et en Angleterre au Moyen Âge ", Homenaje a Jaime Vicens Vives, éd. J. Maluquer de Motes, Barcelone, 1965-1967 (2 vol.), t. I, p. 571-572, et R. Davis, «The Warhorses of the Normans ", Anglo-Norman Studies. Proceedings of the Battle Conference, éd. R. Allen Brown, Woodbridge, 1987, p. 73.

${ }^{72}$ A. Forey, The Templars in the Corona de Aragón, Londres, 1973, p. 324.

${ }^{73}$ Les rares éléments susceptibles d'être rassemblés à ce sujet, en particulier à l'Archivo Histórico Nacional, intéressent exclusivement l'ordre de l'Hôpital (Ph. Josserand, Église et pouvoir dans la péninsule Ibérique. Les ordres militaires dans le royaume de Castille (12521369), Madrid, Casa de Velázquez, 2004, p. 598).

${ }^{74}$ Cronaca del Templare di Tiro (1243-1314), éd. L. Minervini, Naples, Liguori Editore, 2000, p. 142 : «Fu fait maistre frere Guillaume de Biaujeu, quy fu mout gentil home [...] Et quant il fu fait maistre il estoit en Poille comandour, et demoura outremer .ii. ans, visita toutes les maysons dou Temple au royaume de France, d'Engleterre et d'Espaigne, et amasa grant trezor et vint à Acre ». 
recueillir ces fonds à l'intention de la Terre sainte dans le transport desquels plusieurs frères castillans avaient acquis une telle maîtrise qu'ils furent précocement associés à la collecte de la décime pontificale ${ }^{75}$.

Appuyer l'Orient latin, c'était non seulement lui fournir des ressources et de l'argent, mais aussi lui envoyer des combattants afin de faire face à l'Islam ${ }^{76}$. À de tels contingents, les Templiers castillans sont tenus dans la critique pour n'avoir eu aucune part ${ }^{77}$. Pourtant, au tournant des $\mathrm{XIII}^{\mathrm{e}}$ et $\mathrm{XIV}^{\mathrm{e}}$ siècles, plusieurs frères de la province connurent après être tombés aux mains des Mamelouks l'expérience des geôles égyptiennes ${ }^{78}$, où Reddecœur s'était probablement éteint ${ }^{79}$. Prisonnier depuis 1305 au moins ${ }^{80}$, Vasco Pérez Fajardo, issu d'un lignage de petite noblesse

\footnotetext{
${ }^{75}$ Signalé par G. Martínez Díez, Los Templarios en la Corona de Castilla, Burgos, 1993, p. 44, un document publié par D. Mansilla, La documentación pontificia de Honorio III (1216-1227), Rome, 1965, p. 287-288, doc. 387, fait état en 1221 de l'investissement de Diego Manso, attesté trois ans plus tôt comme commandeur de Ponferrada (A. Quintana Prieto, Tumbo viejo de San Pedro de Montes, León, 1971, p. 386, doc. 283), dans les opérations de collecte organisées en Castille, dont Rome avait largement chargé les Templiers, ainsi que l'a souligné A. Rodríguez López, La consolidación territorial de la monarquía feudal castellana. Expansión y fronteras durante el reinado de Fernando III, Madrid, 1994, p. 104.

${ }^{76}$ Pour le Temple, ce principe a été rappelé par A. Forey, « Towards a Profile of the Templars in the Early-Fourteenth Century », The Military Orders. Fighting for the Faith and Caring for the Sick, éd. M. Barber, Aldershot, 1994, p. 201, et The Fall of the Templars in the Crown of Aragon, Aldershot, 2001, p. 83.

77 Signalé par $\mathrm{Ph}$. Josserand, Église et pouvoir dans la péninsule Ibérique. Les ordres militaires dans le royaume de Castille (1252-1369), Madrid, Casa de Velázquez, 2004, p. 598-599, ce fait relève d'une longue tradition remontant pour le moins à M. Fernández de Navarrete, Españoles en las cruzadas, Madrid, 1816, rééd. Madrid, 1986, p. 35, qui, parmi les frères de l'ordre présents en Orient, s'est uniquement attaché aux Catalans et aux Portugais.

${ }^{78}$ Sans référence à des frères castillans, la captivité des Templiers au Caire a été analysée par A. Forey, "The Military Orders and the Ransoming of Captives from Islam (Twelfth to Early-Fourteenth Century) », Studia Monastica, 33, 1991, p. 262-264, repris dans Id., Military Orders and Crusades, Aldershot, 1994, VI, et P.-V. Claverie, «Le statut des Templiers capturés en Orient durant les croisades », La liberazione dei captivi tra Cristianità e Islam. Oltre la crociata e il gihad: tolleranza e servizio umanitario, éd. G. Cipollone, Cité du Vatican, 2000, p. 509-511.

${ }^{79}$ En 1311, la déposition du frère sergent Tomás de Pamplona présente comme mort le digniatire qui l'avait jadis reçu au Temple (J. Michelet, Le procès des Templiers, Paris, Éditions du C.T.H.S., 1987 [2 vol.], t. II, p. 16).

${ }^{80}$ Le fait ressort d'une lettre de Jacques II d'Aragon, conservée à l'Arxiu de la Corona d'Aragó et publiée en partie par A. S. Atiya, Egypt and Aragon. Embassies and Diplomatic Correspondence between 1300 and 1330 A.D., Leipzig, 1938, p. 27 : «Et com nos siam estaz pregats pel noble et car nabot nostre don Ferrando, rey de Castela, que deiam la vostra noblea pregar per Johan Pereç Calvets et Vasco Periç Fajardo et Alfonso Peris, que son en vostre poder, los quals son de ça terra ».
} 
galicienne alors en passe de s'affirmer à Murcie ${ }^{81}$, restait encore détenu treize ans plus tard, à la différence de son compagnon d'infortune, Alfonso Pérez, dont on ignore le destin ${ }^{82}$. À l'époque, des Templiers castillans semblent avoir résidé à Chypre assez régulièrement, parmi lesquels plusieurs accédèrent à un certain degré de responsabilité, s'intégrant le cas échéant au couvent de Jacques de Molay, tels le trésorier de l'ordre Martín López et le commandeur de la Voûte Guillermo de Orense en $1292^{83}$. À ces frères qui ont appartenu à l'entourage magistral, on peut ajouter Domingo, utilisé en 1304 comme messager ${ }^{84}$, ou Martín Martínez qui, arrivé

\footnotetext{
${ }^{81}$ À partir de l'étude de J. Torres Fontes, «Los Fajardo en los siglos XIV y XV », Miscelánea Medieval Murciana, 4, 1978, p. 107-178, surtout p. 110-121, le fait a été rappelé par D. Menjot, Murcie castillane. Une ville au temps de la frontière (1243-milieu du XV $V^{e}$ siècle), Madrid, Casa de Velázquez, 2002 (2 vol.), t. II, p. 984-985.

${ }^{82}$ Á. Masiá de Ros, La Corona de Aragón y los Estados del Norte de África. Política de Jaime II y Alfonso IV en Egipto, Ifriquía y Tremecén, Barcelone, 1951, p. 313, doc. 41 : «En aquest cas los misatges demanen que almenys lo solda vulla absolre e deliurar tots aquels que son en so poder dels regnes e de les terres del rey d'Arago, e encara almenys tres qui no son de la sua terra, ço es a saber Bertran de la Popia e Felip de Menalef de Xipre e Vasco Fagardo d'Ortiguera, qui fo templer, e Gorart de la Perosa, qui fo templer, per los quals alcuns amics lurs qui an servit al rey d'Arago li han suplicat quen degues pregar lo solda ». P.-V. Claverie, « La Cristiandat en mayor peril ou la perception de la question d'Orient dans la Catalogne de la fin du XIII ${ }^{\mathrm{e}}$ siècle », Les Templiers en pays catalan, éd. R. Vinas et al., Perpignan, 1997, p. 116, n. 97, a identifié à juste titre le frère Vasco Fajardo d'Ortiguera au Vasco Pérez détenu en 1305 et lui a reconnu une origine castillane, qu'en revanche on ne saurait prêter à Gérard de Lapeyrouse, né d'un lignage auvergnat, ainsi que l'a fait valoir récemment l'auteur en corrigeant sa position initiale (Id., «Apud Ciprum Nicossiam : notes sur les relations cyprioauvergnates au XIII ${ }^{\mathrm{e}}$ siècle », Epethrida ton Kentron Epistimonikôn Ereunôn, 31, 2005, p. 6869).

${ }^{83}$ Sur la base d'une lettre du grand-maitre publiée par A. Forey, The Templars in the Corona de Aragón, Londres, 1973, p. 405-406, doc. 36, P.-V. Claverie, L'ordre du Temple en Terre sainte et à Chypre au XIII siècle, Nicosie, 2005 (3 vol.), t. I, p. 210, et t. II, p. 330, 334, a identifié ces deux frères derrière les graphies françaises de Martin de Loup et de Guillem d'Ourenç ; le même auteur (ibid., t. I, p. 209, et t. II, p. 338) a fait l'hypothèse qu'en 1271 déjà, le maréchal de l'ordre était castillan, proposant de voir en Cimbelardus un frère du nom de Sancho Belardo, mais, il y a peu, J. Burgtorf, «The Templars' and Hospitallers' High Dignitaries: Aspects of International Mobility », International Mobility in the Military Orders (XII th to $X V^{\text {th }}$ Centuries): Travelling on Christ's Business, éd. J. Burgtorf et H. Nicholson, Cardiff, 2006, p. 14, l'a pour sa part identifié à Amblard de Vienne, un frère provençal.

${ }^{84}$ Mentionné dans une lettre de Pere de Castelló à Pere de Sant-Just, éditée par H. Finke, « Nachträge und Ergänzungen zu den Acta Aragonensia (I-III) », Spanische Forschungen der Görresgesellschaft, 4, 1933, p. 445-446, doc. 9, ce frère a été identifié par A. Demurger, Jacques de Molay. Le crépuscule des Templiers, Paris,Payot, $2007^{2}$, p. 232-233, sur la base de l'analyse d'un document auquel, sans évoquer Domingo, s'était précédemment attaché J. Burgtorf, «Leadership Structures in the Orders of the Hospital and the Temple (Twelfth to Early-Fourteenth Century). Selected Aspects », The Crusades and the Military Orders. Expanding the Frontiers of Medieval Latin Christianity, éd. Z. Hunyadi et J. Laszlovszky, Budapest, 2001, p. 386.
} 
sur l'île l'année suivante ${ }^{85}$, apparaît suffisamment connu lors de l'interpellation de 1310 pour que des nobles orientaux, comme Raymond de Bentho et Balian de Montgisard, soient invités à s'exprimer sur sa réputation ${ }^{86}$. Au début du XIV ${ }^{\mathrm{e}}$ siècle, la présence de Templiers castillans en Orient procède d'une tradition plus ancienne qu'on pourrait a priori le croire. Le premier passage documenté du supérieur provincial, au début des années 1230, est celui dont prit l'initiative Esteban de Belmonte, maître dans les trois royaumes ibériques occidentaux ${ }^{87}$, après avoir réuni un chapitre à Mayorga ${ }^{88}$. Il est probable que des frères issus de l'Occident

\footnotetext{
${ }^{85}$ Bertrand de Brandisio, interrogé à Chypre en mai 1310, dit avoir intégré l'ordre à Marseille cinq ans plus tôt, en présence du frère castillan (K. Schottmüller, Die Untergang des Templerordens, Berlin, 1887 [2 vol.], t. II, p. 208, et A. Gilmour-Bryson, The Trial of the Templars in Cyprus. A Complete English Edition, Leyde, Brill, 1998, p. 139).

${ }^{86}$ Publiés par K. Schottmüller, Die Untergang des Templerordens, Berlin, 1887 (2 vol.), t. II, p. 157 et 163, et traduits par A. Gilmour-Bryson, The Trial of the Templars in Cyprus. A Complete English Edition, Leyde, Brill, 1998, p. 62 et 73, leurs témoignages, comme ceux de l'ensemble des laïcs alors interrogés, sont favorables aux Templiers (P. Edbury, «The Templars in Cyprus » et A. Gilmour-Bryson, «Testimony of Non-Templar Witnesses in Cyprus ", The Military Orders. Fighting for the Faith and Caring for the Sick, éd. M. Barber, Aldershot, Ashgate, 1994, p. 205). Néanmoins, Martín Martínez a fait l'objet d'accusation de violences sexuelles de la part du frère sergent Pierre de Safed, interrogé en octobre 1307 à Paris après avoir été sans doute torturé : " unde dixit per juramentum suum quod contingit cum quadam nocte exiret de camera dicti magistri quidam frater dicti ordinis Yspanus, qui vocabatur Martinus Martini, vocavit eum et abusus fuit eo carnaliter» (J. Michelet, Le procès des Templiers, Paris, Éditions du C.T.H.S., 1987 [2 vol.], t. II, p. 293).

${ }^{87}$ G. Martínez Díez, Los Templarios en la Corona de Castilla, Burgos, Editorial La Olmeda, 1993, p. 65, et J. Valente, «Soldiers and Settlers. The Knights Templar in Portugal, 1128$1319 »$, Thèse de doctorat, University of California, 2002, p. 185, le tiennent pour castillan, alors que C. de Ayala Martínez, «Fernando III y las órdenes militares », Fernando III y su tiempo, 1201-1252. Congreso de estudios medievales, Ávila, 2003, p. 83, et D. Carraz, L'ordre du Temple dans la basse vallée du Rhône, 1124-1312. Ordres militaires, croisades et sociétés méridionales, Lyon, Presses Universitaires, 2005, p. 319, le rattachent l'un au Languedoc, l'autre à la Catalogne, le premier de ces auteurs revenant par là sur sa position antérieure (C. de Ayala Martínez, «Frontera castellano-leonesa y órdenes militares. Problemas de jurisdicción [siglos XII-XIII] », Jornadas de cultura hispano-portuguesa, éd. V. Á. Álvarez Palenzuela, Madrid, 1999, p. 67 et 69).

${ }^{88}$ L'engagement du maître en Terre sainte ressort d'un contrat agraire qu'il souscrivit le 13 janvier 1232 «cum consilio de ipso comendatore qui est in bauilia d'Amoeyro domnus Fernandus Salvatoris et per consilium capituli generali quod fuit factum in Mayorga quando iebam me pera oltramar» (M. Romaní Martínez, Colección diplomática do mosteiro cisterciense de Santa Maria de Oseira [Ourense], 1025-1399, Saint-Jacques de Compostelle, 1989 [3 vol.], t. I, p. 335-337, doc. 349). De Mayorga, il est probable que le dignitaire du Temple se soit attaché à réunir une part des ressources en vue de son passage si l'on s'en tient à l'opinion de P. Martínez Sopena, La Tierra de Campos occidental. Poblamiento, poder y comunidad del siglo X al XIII, Valladolid, 1985, p. 173-176, qui signale que dans cette ville, en plein développement dans la première moitié du XIII ${ }^{\mathrm{e}}$ siècle, les maisons des ordres
} 
péninsulaire s'étaient alors déjà battus en Terre sainte ${ }^{89}$. D'autres en tout cas n'allaient guère tarder à s'y engager, accomplissant parfois, à l'instar de leurs homologues hospitaliers ${ }^{90}$, de belles carrières, tels Fernán Español, commandeur du Temple d'Antioche en $1249^{91}$, ou Gonzalo Martín, en charge treize ans plus tard de la maison de l'ordre dans la ville d'Acre ${ }^{92}$.

C'est seulement au hasard de la documentation, comme Damien Carraz vient de le rappeler pour la basse vallée du Rhône, qu'on peut identifier les Templiers hors de leur région d'origine ${ }^{93}$. À cette règle, la Castille ne fait en rien exception. En comparaison de leurs coreligionnaires français et aragonais, les frères issus de cette province qui ont lutté dans l'Orient latin sont sans conteste moins nombreux, mais

« sirven como centro preceptor de las rentas de respectivas instituciones en un ámbito más o menos extenso que se dilata hasta Valdescorriel, Aguilar de Campos o Villagómez ».

${ }^{89}$ Sans doute est-ce le cas du frère Thomas, mentionné par A. Demurger, Les Templiers. Une chevalerie chrétienne au Moyen Âge, Paris, Éditions du Seuil, 2005, p. 337 et 375, qui, avec son bateau, s'est mis au service du roi d'Angleterre Henri III durant la guerre avec la France qui s'est déroulée entre 1224 et 1226. Mise au jour par H. Nicholson, «The Military Orders and the Kings of England in the Twelfth and Thirteenth Centuries », From Clermont to Jerusalem. The Crusades and Crusader Societies (1095-1500), éd. A. Murray, Turnhout, 1998, p. 212-215, l'existence de ce personnage, attestée par plusieurs documents extraits des Patent Rolls du Public Record Office, invite à tenir pour probable l'engagement en Terre sainte de Templiers issus des royaumes hispaniques occidentaux dès l'époque de la cinquième croisade. En effet, dépeint comme espagnol par l'historienne britannique (ibid., p. 212), Thomas est plus exactement originaire de Castille, du León ou du Portugal car le maître provincial auquel Henri III a payé ses services, un certain P. dans les actes anglais, est en réalité Pedro Alvítiz, supérieur de l'ordre dans les trois royaumes occidentaux de la Péninsule entre 1216 et 1227 . Tenu pour « a skilled sailor who could be relied upon » (ibid., p. 215), le frère n'a pas pu acquérir sa connaissance navale et son aptitude au commandement dans l'Occident ibérique, où, à la différence de l'Orient méditerranéen, le Temple ne s'était pas encore battu sur mer.

${ }^{90}$ Plusieurs frères de l'Hôpital originaires des royaumes hispaniques occidentaux ont fait carrière en Orient, à l'instar du drapier Martín Sánchez, attesté en 1248, ou du maréchal Rodrigo Pérez, mentionné en 1271 en compagnie de Rodrigo Rodríguez, futur hospitalier de l'ordre (J. Delaville le Roulx, Cartulaire général de l'ordre des Hospitaliers de Saint-Jean de Jérusalem, Paris, 1894-1906 [4 vol.], t. II, p. 673-675, doc. 2482, et t. III, p. 259-260, doc. 3433, et p. 300-301, doc. 3519).

${ }^{91}$ M.-L. Bulst-Thiele, Sacrce domus militice Templi Hierosolymitani magistri. Untersuchungen zur Geschichte des Templerordens (1118/19-1314), Göttingen, 1974, p. 231.

${ }^{92}$ En mai 1262, il figure comme témoin dans deux documents conclus en Acre (Bibljoteca Nazzjonali, Scritture Originali, vol. 18, doc. 2 et 3, publ. J. Delaville le Roulx, Cartulaire général de l'ordre des Hospitaliers de Saint-Jean de Jérusalem, Paris, 1894-1906 [4 vol.], t. III, p. 30-31 et 31-33, doc. 3028 et 3029), et il est mentionné dans un acte du 18 décembre suivant (Bibljoteca Nazzjonali, Scritture Originali, vol. 18, doc. 4, cit. M.-L. Bulst-Thiele, Sacrce domus militice Templi Hierosolymitani magistri. Untersuchungen zur Geschichte des Templerordens [1118/19-1314], Göttingen, 1974, p. 257).

${ }^{93}$ D. Carraz, L'ordre du Temple dans la basse vallée du Rhône, 1124-1312. Ordres militaires, croisades et sociétés méridionales, Lyon, Presses Universitaires, 2005, p. 323. 
leur contingent, loin d'être négligeable, révèle des échanges variés entre l'Occident péninsulaire et la Terre sainte, invalidant l'image colportée à l'ordinaire de combattants uniquement soucieux de la dimension hispanique de leur engagement au service de la foi chrétienne.

D'un tel parcours, nécessairement rapide, il ressort que les Templiers castillans, en dépit de ce que l'on peut encore affirmer ${ }^{94}$, se sont investis dans la défense des États latins d'Orient et même qu'ils l'ont fait en nombre passé le milieu $\mathrm{du} \mathrm{XIII}^{\mathrm{e}}$ siècle, une fois les Mamelouks en situation de se défaire de la menace croisée. Certes, pour une bonne part, la trace de leur engagement militaire s'est perdue, mais elle n'a pas pour autant entièrement disparu. Les preuves qu'il m'a été donné de réunir demandent à être complétées. Bien des fonds d'archives restent à interroger dans lesquels tout porte à croire que d'autres éléments sommeillent, qui parfois ont été évoqués dans des travaux d'érudition anciens ${ }^{95}$, et des supports de tout autre nature, surtout, requièrent d'être pris en compte, textuels sans doute ${ }^{96}$, mais également matériels ${ }^{97}$. Une enquête très large mériterait donc d'être mise en

\footnotetext{
${ }^{94}$ J. Clemente Ramos et J. L. de la Montaña Conchiña, « Las órdenes militares en el marco de la expansión cristiana de los siglos XII-XIII en Castilla y León : la orden del Temple en Extremadura ", E-Spania. Revue électronique d'études médiévales hispaniques, 1 (2005), p. 1-25, reprennent une fois de plus l'idée d'une autonomie du Temple en Castille par rapport à l'Orient latin, lequel n'est à aucun moment évoqué. L'ouvrage de divulgation de G. Martínez Díez, Los Templarios en los reinos de España, Madrid, 2001, présente le même défaut.

${ }^{95} \mathrm{Qu}$ 'il suffise en guise d'exemple de signaler les éléments rapportés au début du XIX ${ }^{\mathrm{e}}$ siècle par Ph. Grouvelle, Mémoires historiques sur les Templiers, Paris, [s.d.], rééd. Paris, Éditions Jean de Bonnot, 2004, p. 28 et 258, à partir de l'ouvrage de F. Münter, Statuten Buch des Ordens der Tempelherren, Berlin, 1794, reposant sur certaines sources inédites ou aujourd'hui disparues.

${ }^{96}$ Il en est ainsi en particulier de La Gran Conquista de Ultramar. Biblioteca Nacional Ms. 1187, éd. L. Cooper, Madison, 1989, dont, il y a près de deux siècles, M. Fernández de Navarrete, Españoles en las cruzadas, Madrid, 1816, rééd. Madrid, 1986, p. 26, a rapporté que le texte attribuait à un émir damascène l'intention de confier ses enfants à " un caballero de España, miembro del Temple », dont il aurait été «prendado del valor y virtud ».

${ }^{97}$ Pour la couronne d'Aragon et le royaume de Portugal, d'intéressants rapprochements entre l'architecture templière en Orient et la construction militaire locale ont été réalisés à l'initiative de J. Fuguet i Sans, «Els castells templers de Gardeny i Miravet i el seu paper innovador en la poliorcètica i l'arquitectura catalanes del segle XII », Acta Historica et Archoelogica Medicevalia, 13 (1992), p. 353-374, et «De Miravet (1153) a Peníscola (1294): novedad y persistencia de un modelo de fortaleza templaria en la provincia catalanoaragonesa de la orden », Acri 1291. La fine della presenza degli ordini militari in Terra Santa e i nuovi orientamenti nel XIV secolo, éd. F. Tommasi, Pérouse, 1996, p. 43-67, et de M. J. Barroca, «A ordem do Templo e a arquitectura militar portuguesa do século XII », Portugalia, 17-18 (1996-1997), p. 171-209. Il serait à souhaiter qu'une approche similaire soit
} 
place dans l'esprit des recherches actuelles sur la mobilité au sein des ordres militaires ${ }^{98}$. Du lancement d'un tel projet, sans vouloir aucunement préjuger de ses résultats, peut-être pourrait-on espérer que l'histoire du Temple en Castille cesse enfin d'être écrite sans référence à la Terre sainte.

Philippe Josserand Université de Nantes - CRHIA - EREMAM

développée à l'échelle de la Castille et du León, qui souffrent pour le Temple en matière d'architecture militaire d'un déficit historiographique qu'achève de rappeler très justement J. Fuguet i Sans, «La historiografía sobre arquitectura templaria en la Península Ibérica », Anuario de Estudios Medievales, 37 (2007), p. 386.

${ }^{98}$ International Mobility in the Military Orders (Twelfth to Fifteenth Centuries): Travelling to Christ's Business, éd. J. Burgtorf et H. Nicholson, Cardiff, 2006. 